\title{
USO DE IMAGENS DO SENSOR MODIS PARA IDENTIFICAÇÃO DA SECA NA CANA-DE- AÇÚCAR ATRAVÉS DE ÍNDICES ESPECTRAIS
}

\author{
Sugarcane drought assessment using spectral indices from MODIS sensor images
}

\author{
Daniel Garbellini Duft ${ }^{1 *}$; Michelle Cristina Araujo Picoli ${ }^{2}$
}

\author{
${ }^{1}$ Analista; Laboratório Nacional de Ciência e Tecnologia do Bioetanol;*daniel.duft@ctbe.cnpem.br \\ *Autor para correspondência \\ 2Pesquisadora, Instituto Nacional de Pesquisas Espaciais; mipicoli@gmail.com
}

Artigo enviado em 19/07/2017, aceito em 23/12/2017 e publicado em 10/04/2018.

Resumo - A cana-de-açúcar é uma cultura de grande importância econômica para o mundo, principalmente para o Brasil, onde representa 15\% do PIB agrícola. Com as mudanças climáticas globais, alguns fenômenos extremos tendem a acontecer com mais frequência, entre eles, a seca que tem castigado várias culturas agrícolas. Apesar de ser uma planta resistente a seca, a ocorrência causa déficits de produtividade na cana-de-açúcar, causando enormes prejuízos aos produtores e um desequilíbrio no mercado de açúcar e combustíveis. Para monitorar eventos de seca diversos índices e métodos, baseados em dados climáticos ou em imagens espectrais, foram criados, porém nenhum deles é específico para a cultura da cana-de-açúcar. O trabalho testou alguns índices espectrais do sensor MODIS capazes de monitorar de eventos de seca para a cana-de-açúcar. Esta avaliação foi realizada em duas etapas: uma temporal e uma espacial. Os índices espectrais foram comparados com o SPI calculado para 1 e 3 meses. Foi observado que os índices espectrais que melhor monitoram eventos de seca na cana de açúcar são o GVMI, NDWI e SRWI. Índices espectrais que não tiveram correlação com eventos de seca foram: o MSI e NDI7.

Palavras-chave - Imagem de satélite. SPI. Análise espacial.

\begin{abstract}
Sugarcane has great economic importance to the world, especially to Brazil, where it represents $15 \%$ of agricultural GDP. The global climate change makes some extreme phenomena tend to happen more often, among them, the drought that has punished several agricultural crops. Despite being a drought resistant plant, the occurrence causes productivity deficits in sugarcane, causing huge losses to producers and an imbalance in the sugar and fuels market. To monitor drought events, several indices and methods, based on climatic data or spectral images, were created, but none of them are specific to the sugarcane crop. The paper tested some indexes of MODIS sensor, able to monitor drought events in sugarcane. This evaluation was carried out in two stages: time and space. The spectral indices were compared with the SPI calculated for 1 and 3 months. It has been observed that the best spectral indexes to sugarcane are GVMI, NDWI and SRWI. Spectral indexes that did not correlate with drought events: MSI and NDI7.
\end{abstract}

Keywords - Satellite images. SPI. Spatial analysis.

\section{INTRODUÇÃO}

O Brasil é o maior produtor de cana-deaçúcar do mundo (FAO, 2016), sendo que na safra 2015/2016 atingiu uma produção recorde de 665 milhões de toneladas, com $55 \%$ da produção total destinada a fabricação de etanol, chegando a 30,4 milhões de metros cúbicos (CONAB, 2016). O etanol de cana-de-açúcar é produzido no país desde o início do século XX, porém foi com incentivo governamental, através do PROALCOOL, nos anos 70 que houve um aumento na produção que durou até meados dos anos 90 (WALTER et al., 2011). Com a inserção dos veículos bicombustíveis na frota nacional houve um novo aumento a partir de 2003 que durou até 2009 (MACHADO et al., 2014). A matéria prima do etanol, a cana-de-açúcar é uma cultura que traz grandes benefícios econômicos para o Brasil, a parcela do Produto Interno Bruto (PIB) da atividade sucroenergética foi de 108 bilhões de reais em 2015, isso representou quase $15 \%$ de todo PIB agrícola do país (CEPEA, 2016). Além disso, esse valor foi $20 \%$ 
maior que o PIB da cadeia da soja, que é a cultura com maior área cultivada no país.

O aumento da área destinada a produção de cana-de-açúcar, puxado pelo aumento na demanda por biocombustíveis, foi de aproximadamente 4,5 Mha entre os anos de 2000 e 2014 em todo território brasileiro (IBGE, 2016). Porém, a produtividade dos canaviais tem decrescido desde 2008 por diversos fatores como clima, manejo inadequado e dificuldades financeiras do setor (WALTER et al., 2013).

Sabe-se que o crescimento dos colmos da cana-de-açúcar está altamente ligado a ausência de déficit hídrico no solo (KOEHLER et al., 1982), e que para as plantas terem um crescimento significativo precisam de água a disposição todo o tempo, e que períodos de seca podem ser um grande problema para seu desenvolvimento pleno (DOORENBOS e KASSAM, 1979). Contudo, estudos comprovam que nas últimas décadas em decorrência do aumento de temperatura, os períodos de seca tendem a ser mais recorrentes, uma vez que as mudanças climáticas globais aumentam a incidência de eventos extremos (PALMER, 2002; ORLOWSKY e SENEVIRATNE, 2011; ZSCHEISCHLER et al., 2014; IPCC, 2014). Portanto, a variação nos padrões espaciais da precipitação, para a agricultura não irrigada, como é o caso da cana-de-açúcar no Brasil, podem causar a perda de produtividade e até mesmo quebras de safra (MARIN et al., 2012). A seca que ocorreu em 2014 no centro-sul do país prejudicou diversas culturas agrícolas, segundo a UNICA (2016) a cana-de-açúcar teve uma redução de $5 \%$ na produção além de impactos na safra seguinte. Em valores monetários essa perda chegou perto de 2 bilhões de reais.

Devido aos prejuízos e as preocupações que de eventos de secas sejam cada vez mais recorrentes, há a necessidade de se conhecer e a até prever esse fenômeno. Para isso, alguns índices foram criados para monitorar eventos de seca meteorológica como o Palmer Drought Severity Index (PDSI) (PALMER, 1965), que identifica a seca em um intervalo temporal em que a precipitação está aquém daquela considerada a normal, porém para o cálculo deste índice é necessária uma série histórica de precipitação, de evapotranspiração e temperatura média do ar. Além do PDSI, existe o Standardized Precipitation Index (SPI) (MCKEE et al., 1993 e MCKEE et al., 1995). O SPI utiliza apenas informações de precipitação e compara valores atuais com a distribuição da série histórica através de um processo de padronização, por conta disso, seu uso é amplamente disseminado e os resultados são utilizados para monitorar eventos de seca ao longo do planeta. O SPI é ainda bastante utilizado em plataformas de monitoramento de seca. Nos Estados Unidos, o The National Drought
Mitigation Center (NDMC) e United States Department of Agriculture (USDA) Federal Drought Assistance utilizam o SPI para monitoramento da seca no país, tanto voltado para o monitoramento de desastres como para agricultura. No Brasil o projeto Infoseca do Instituto Agronômico de Campinas (IAC) também utiliza o SPI para detectar eventos de seca.

Mas, além de monitorar os eventos de seca meteorológica, é de extrema importância verificar a implicação dessa seca na vegetação (seca agrícola). O sensoriamento remoto é uma ferramenta que consegue extrair atributos meteorológicos, características biofísicas e detalhes do terreno, e cada vez mais tem sido usado para monitoramento de secas. Son et al. (2012) e Wu et al. (2015) utilizaram sensoriamento remoto para monitorar grandes áreas agrícolas e ressaltaram a importância de ter-se uma informação em tempo quase real para o planejamento e tomada de decisões do governo e dos produtores. Para a cana-deaçúcar que é uma cultura plantada em larga escala e ocupa aproximadamente 10 milhões de hectares só no Brasil (UNICA, 2016), o monitoramento de eventos de seca via sensoriamento remoto é essencial para prevenir e alertar grandes perdas na produção, como a ocorrida em 2014.

Os dados provenientes de sensoriamento remoto podem ser usados isoladamente ou em conjunto com dados locais para identificar e mensurar eventos de seca (RHEE et al., 2010). Para dados meteorológicos, além de utilizar-se de dados de estações para o cálculo do SPI e do PDSI, também é possível utilizar-se dados provindos de modelos, como por exemplo o European Centre for Medium-Range Weather Forecasts (ECMWF). O ECMWF é um modelo de assimilação que conta com dados de diversas fontes e traz parâmetros climáticos com $0,25^{\circ}$ de resolução espacial (DEE et al., 2011).

Além dos dados meteorológicos e dos índices calculados através desses dados há os índices espectrais que são calculados utilizando imagens de sensoriamento remoto. Os índices espectrais são ferramentas essenciais para a caracterização de alvos, eventos e condições dos mesmos. O primeiro índice espectral a ter implicação em agricultura foi o Normalized Difference Vegetation Index (NDVI) desenvolvido por Tucker (1979), que foi desde então amplamente utilizado para todas as culturas agrícolas em todas as regiões do globo. Porém, outros índices espectrais têm sido criados e aplicados com sucesso para o monitoramento da seca na agricultura. Zhang et al. (2015) aplicaram o Normalized Difference Water Index (NDWI) e o Simple Radio Water Index (SRWI) para o trigo na China. Nagler et al. (2013) usaram o Global Vegetation Moisture Index (GVMI) e o Ehanced Vegetation Index (EVI) para monitorar 
evapotranspiração de culturas ao longo do rio Colorado nos EUA. Por sua vez Mamadou et al. (2014) testaram diversos índices para monitorar vegetação nativa no Senegal como o Normalized Difference Infrared Index (NDII), o NDWI, o SRWI e o Moisture Stress Index (MSI). Todos os índices desempenharam bem suas funções para as culturas estudadas, mas poucos desses índices foram aplicados para a cultura da na cana-de-açúcar até agora, um exemplo que temos é Adami et al. (2012) que usa o EVI2 para identificar séries temporais e mudança de uso do solo, mas nenhum estudo foi feito para o monitoramento da seca.

Por existirem diversos índices espectrais que identificam aspectos da seca na vegetação, é necessário um trabalho para testar o potencial desses índices para monitorar a seca na cana-de-açúcar. Desta forma, o objetivo desse trabalho foi testar a eficiência dos índices de seca mais utilizados com imagens do sensor MODIS (Moderate Resolution Imaging Spectroradiometer) para monitorar eventos de seca em cana-de-açúcar no estado de São Paulo para quatro safras. O sensor MODIS foi escolhido por ser o que apresenta melhor resolução temporal, um número de bandas suficiente para os cálculos dos índices espectrais e por ter distribuição gratuita. Além disso, o sensor MODIS é integrante do programa Earth Observing System (EOS) da NASA que prevê continuidade em satélites e sensores de monitoramento da Terra. Sendo assim, esse estudo cria uma base para que possam ser usadas outras imagens com as características semelhantes.

\section{MATERIAL E MÉTODOS}

Área de estudo

A área de estudo foi o estado de São Paulo. O estado de São Paulo (Figura 1) é o maior produtor de cana-de-açúcar do Brasil e na safra 2014/15 possuía uma área plantada de 5,6 milhões de hectares (IBGE, 2016). O clima segundo a classificação de Strahler é tropical e subtropical úmido, com temperaturas variando em torno de mais de $22^{\circ} \mathrm{C}$ no verão e maior que $10^{\circ} \mathrm{C}$ no inverno (PEEL et al., 2007). A precipitação média anual é de $1300 \mathrm{~mm}$, de acordo com os dados do ECMWF para a normal climatológica calculada, o climograma gerado através desses cálculos está na Figura 2. Esses são fatores que segundo Manzatto et al. (2009) são propícios para o cultivo da cana-de-açúcar.

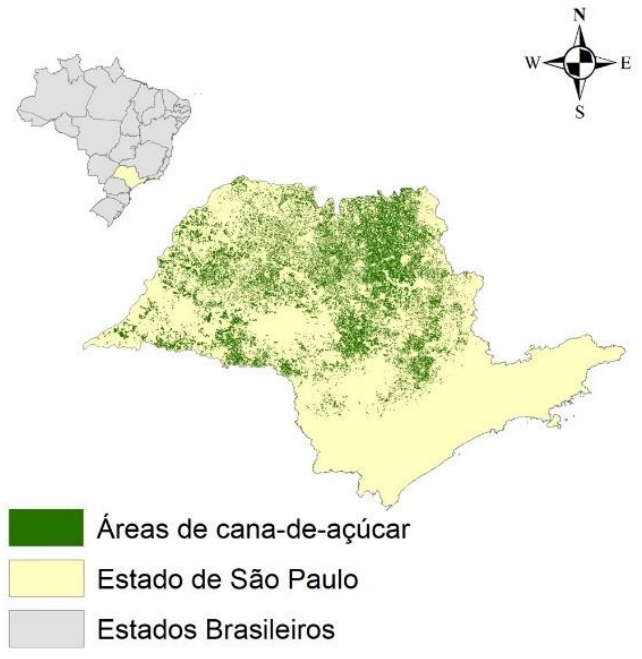

Figura 1: Área de estudo

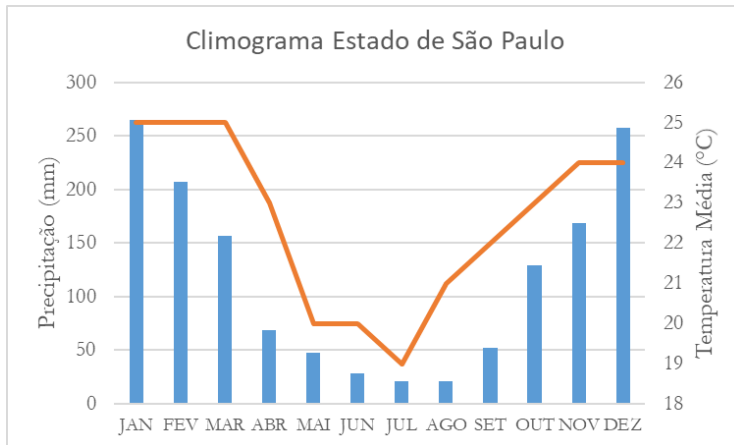

Figura 2: Climograma Estado de São Paulo (ECMWF)

\section{Standardized Precipitation Index (SPI)}

O Standardized Precipitation Index (SPI) foi criado por McKee et al. (1993) e McKee et al. (1995) com o objetivo de identificar e quantificar a intensidade da seca meteorológica.

Alguns autores como Caccamo et al. (2011) e Du et al. (2013) utilizaram o SPI para caracterizar seca. O uso deste índice é prático e eficaz, pois pode apontar eventos de seca meteorológica a partir da série histórica da região estudada.

Para o cálculo do SPI utiliza-se de uma série histórica de precipitação e através de padronizações identifica-se períodos secos e úmidos. Valores próximos a zero são os normais enquanto os mais próximos de 3 são os mais úmidos e os próximos de -3 os mais secos. O SPI é o índice que foi usado neste trabalho para avaliar quais índices espectrais podem ser um indicador de seca agrícola, pois as secas agrícolas são iniciadas por secas meteorológicas. 


\section{Índices de seca espectrais}

Foram selecionados oito índices espectrais (Tabela 1) que de acordo com os trabalhos levantados na literatura identificavam eventos de seca em áreas com vegetação. Esses índices foram calculados para os meses de outubro a abril das safras 2007/08, 2008/09, 2009/10 e 2013/14 (Tabela 1). Foram escolhidas essas safras por terem sido indicadas como seca, chuvosa, normal e anômala respectivamente.

Tabela 1: Índices espectrais utilizados

\begin{tabular}{|c|c|c|}
\hline Índice & Equação & Referência \\
\hline EVI2 & $2,5\left(\frac{B A N D A 2-B A N D A 1}{B A N D A 2+2,4 B A N D A 1+1}\right)$ & $\begin{array}{c}\text { Jiang } \\
(2007)\end{array}$ \\
\hline GVMI & $\frac{(B A N D A 2+0.1)-(B A N D A 6+0,02)}{(B A N D A 2+0.1)+(B A N D A 6+0,02)}$ & Ceccato \\
\hline MSI & $\frac{B A N D A 7}{B A N D A 2}$ & $\begin{array}{l}\text { Hunt e } \\
\text { Rock } \\
(1989)\end{array}$ \\
\hline NDI6 & $\frac{B A N D A 2-B A N D A 6}{B A N D A 2+B A N D A 6}$ & $\begin{array}{c}\text { Hardisky } \\
\text { et al. } \\
\text { (1983) }\end{array}$ \\
\hline NDI7 & $\frac{B A N D A 2-B A N D A 7}{B A N D A 2+B A N D A 7}$ & $\begin{array}{l}\text { Chuvieco } \\
\text { (2002) }\end{array}$ \\
\hline NDVI & $\frac{B A N D A 2-B A N D A 1}{B A N D A 2+B A N D A 1}$ & $\begin{array}{l}\text { Tucker } \\
(1979)\end{array}$ \\
\hline NDWI & $\frac{B A N D A 2-B A N D A 5}{B A N D A 2+B A N D A 5}$ & $\begin{array}{l}\text { Gao } \\
(1996)\end{array}$ \\
\hline SRWI & $\frac{B A N D A 2}{B A N D A 5}$ & $\begin{array}{l}\text { Zarco- } \\
\text { Tejada e } \\
\text { Ustin } \\
(2001)\end{array}$ \\
\hline
\end{tabular}

*Banda 1: 620-670nm | Banda 2: 841-876nm | Banda 5: 12301250nm | Banda 6: 1628-1652nm | Banda 7: 2105-2155nm

\section{Cálculo e Análise}

Foram utilizadas imagens do produto MOD09A, que fornece imagens de refletância de superfície a cada 8 dias, com resolução espacial de 500 metros do sensor MODIS tanto a bordo do satélite Terra como do satélite Aqua, que possui resolução espacial de $500 \mathrm{~m}$ (USGS, 2016). Essas imagens são fruto de uma composição de oito dias, desta forma, a influência pelo recobrimento de nuvens é diminuída se comparadas a imagens diárias.

Inicialmente as imagens foram extraídas através de um algoritmo desenvolvido em linguagem IDL. Devido a resolução espacial das imagens ser baixa (500 metros), foram selecionados somente pixels puros de cana-de-açúcar para o trabalho, ou seja, aqueles pixels do estado de São Paulo que estavam totalmente dentro das áreas de cana-de-açúcar, que foram previamente mapeadas através do uso de imagens do satélite LANDSAT (com resolução espacial de $30 \mathrm{~m}$ ).
Os 8 índices espectrais foram calculados de forma automatizada através de um algoritmo também implementado em linguagem IDL. Foram geradas 3456 imagens de índices espectrais para o período de 2007 a 2014, para os produtos MOD (satélite Terra) e MYD (satélite Aqua).

Em posse dos índices, calculados para todos os pixels puros do estado de São Paulo, foram separados os dados por regiões (critério espacial). Essas regiões foram as mesmas do modelo ECMWF, que possui resolução espacial de $0,25^{\circ}$ (aproximadamente $24,9 \mathrm{~km}$ ). Foi então calculada a mediana dos pixels puros para cada mês estudado, isso foi feito para cada índice que já havia sido calculado. Esse procedimento evitou que houvesse grande interferência de valores extremos, como quando se utiliza a média.

Em seguida foi calculado o SPI com os dados provindos do ECMWF. Foi levantada a série histórica completa desde janeiro de 1989 até abril de 2015. O SPI foi calculado de 2007 a 2014 para dois períodos: mensal (MÊS) e trimestral (3MESES), isso foi feito para os 243 pontos do estado de São Paulo, porém apenas 184 pontos possuíam cana-de-açúcar plantada. O SPI calculado para o período mensal é diferente do calculado para o período trimestral. Como o cálculo é baseado na distribuição gama da normal climatológica, o SPI mensal é capaz de captar desvios da normal para um mês, assim é um grande indicativo de seca meteorológica. Já o SPI trimestral é capaz de captar desvios para períodos de três meses consecutivos na normal, sendo assim é um índice que consegue captar desvios de seca agrícola para culturas perenes e semiperenes (BLAIN e BRUNINI, 2007). Desta forma, as análises foram divididas em duas etapas:

\section{$1^{a}$ Análise - Critério espacial}

Para analisar o efeito espacial dos índices e a seca na cana-de-açúcar foi feita uma análise separada para cada um dos 184 pontos. Nesta análise foi comparado, para cada ponto, os valores do SPI (mensal (MÊS) e trimestral (3MESES)) com os 8 índices espectrais provindos dos sensores MODIS, os produtos MOD e MYD. Inicialmente, foi calculado o teste de normalidade de Shapiro-Wilk, que mostrou que os dados não apresentavam distribuição normal. Sendo assim, foi realizada uma análise de correlação de Spearman entre o SPI MÊS e o SPI 3MESES com os valores das medianas dos índices espectrais de outubro a abril de cada safra.

\section{$2^{\mathrm{a}}$ Análise - Critério Temporal}

Para analisar o efeito temporal da seca para cada ano safra, foram calculadas as médias, de cada um dos índices espectrais e dos SPIs (mensal e trimestral), de todos os 184 pontos para cada um dos meses 
analisados (outubro, novembro, dezembro, janeiro, fevereiro, março e abril). Para cada mês, de cada uma das safras, as médias dos 184 valores do SPI MÊS e SPI 3MESES foram correlacionadas com as médias dos 184 valores de cada índice espectral, através da correlação de Spearman, uma vez que os dados não apresentam normalidade. Foram feitas análises individuais para os anos safras de 2007/08, 2008/09, 2009/10 e 2013/14.

\section{RESULTADOS E DISCUSSÃO}

\section{Standardized Precipitation Index (SPI)}

Com base nos valores de SPI, calculados a partir dos dados do modelo ECMWF, dos 184 pontos com cana-de-açúcar no estado de São Paulo, foi feita uma média para entender o comportamento dos mesmos ao longo dos meses analisados nas safras 2007/2008, 2008/2009, 2009/2010 e 2013/2014. A Figura 3 mostra o SPI calculado para o período mensal e a Figura 4 o SPI calculado para o período de três meses.

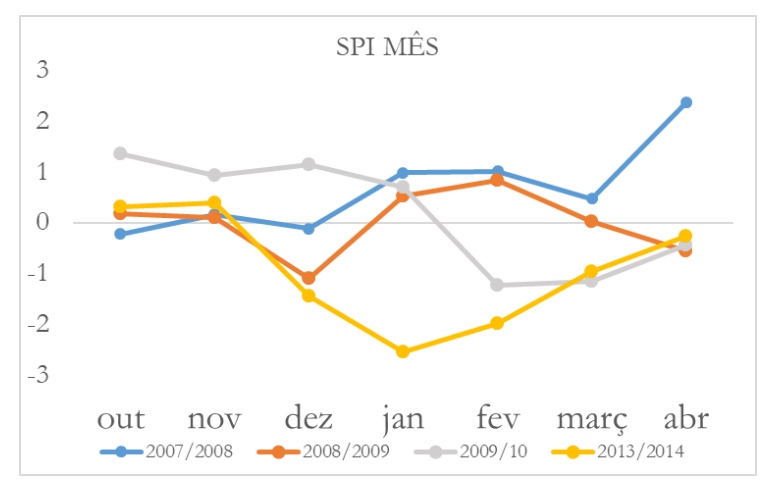

Figura 3: SPI para o período mensal

Os gráficos das Figuras 3 e 4 apresentam valores distintos pois o SPI mensal identifica a situação da precipitação daquele mês em específico, já o SPI trimestral considera aquele mês analisado e os dois meses anteriores a ele, então tem a tendência de suavizar os valores, uma vez que apenas eventos de seca ou chuva em excesso com longa duração são captados pelo mesmo. Conforme apresentado na Figura 3, o ano de 2014 foi o mais seco comparados aos demais, neste ano o estado de São Paulo, bem como o Brasil em geral, sofreu umas das secas mais severas dos últimos anos (GUHA-SAPIR et al., 2014). Ainda assim, em algumas regiões do estado o efeito da seca não foi sentido, mas na média é possível verificar que o comportamento do SPI se manteve abaixo de zero na maior parte do ano de 2014.

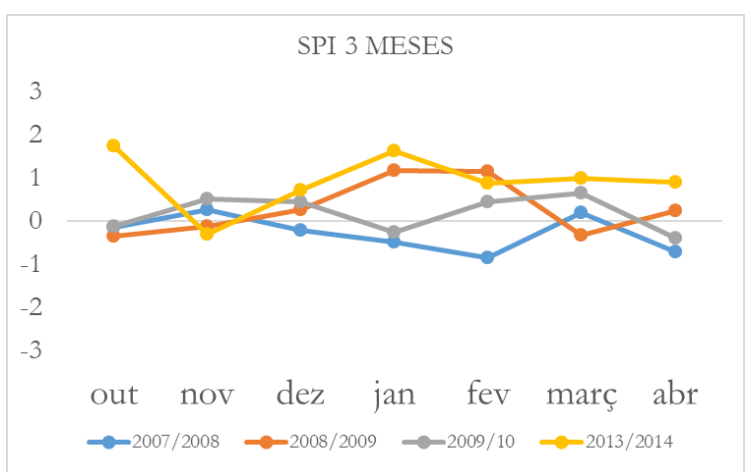

Figura 4: SPI para o período trimestral

Analisando a Figura 3 é possível observar que para o SPI MÊS, o mês de fevereiro apresentou maior distinção entre safras. Sendo que as safras 2007/08 e 2008/09 foram úmidas e as safras 2009/10 e 2013/14 foram secas. Também é possível verificar na Figura 4, no gráfico do SPI trimestral, só que neste gráfico está ao contrário do outro, pois como já observado o SPI trimestral só acusa seca quando esta ocorre nos últimos 3 meses.

Caccamo et al. (2011) estudando as florestas plantadas na Austrália identificou que o melhor mês para analisar correlações com índices espectrais foi o mês de janeiro com o SPI semestral. Já no trabalho de Du et al. (2013) os autores utilizaram o SPI trimestral para correlacionar a seca das culturas de verão em uma província chinesa.

Para identificar a tendência dos índices espectrais em relação ao SPI mensal, foram feitos gráficos de dispersão para cada um dos 8 índices, para o produto MOD e o produto MYD. Para isso, como os valores do SPI mensal variavam entre -3 e 3 e os índices espectrais variavam entre -1 e 1 , com exceção dos índices MSI e SRWI que por serem apenas uma divisão de bandas podem assumir diversos valores. Para corrigir isso, os índices foram normalizados para a mesma escala do SPI (entre -3 e 3) através da Equação 1.

$$
y=\left(6\left(\frac{x-x \text { mín }^{\prime}}{x m a ́ x-x \min ^{\prime}}\right)\right)-3
$$

Onde y indica o valor ajustado, x o valor a ser ajustado, xmín o menor valor do índice e xmáx o maior valor do índice.

Esses gráficos apresentados na Figura 5 e 6 foram confeccionados para o mês de fevereiro de cada uma das safras, por ser um dos meses que apresentaram valores distintos entre safras com predominância de seca e safra com predominância de períodos chuvosos. É possível observar na Figura 5 que nos anos de 2014 e 2010 tanto os valores do índice 
GVMI quanto do índice SPI tiveram valores negativos, ou seja, foi identificado tanto a seca meteorológica pelo SPI quanto a seca na cultura da cana pelo GVMI. Já nos anos de 2009 e 2008 não houve seca meteorológica (valores positivos de SPI), porém a cana apontava que havia deficiência hídrica (valores negativos do índice GVMI). Já no gráfico da Figura 6 é possível observar que o índice NDVI não apontou nenhuma deficiência em nenhuma das safras, em todas as safras os valores foram positivos. Porém, o SPI apontou deficiência hídrica em 2010 e 2014.

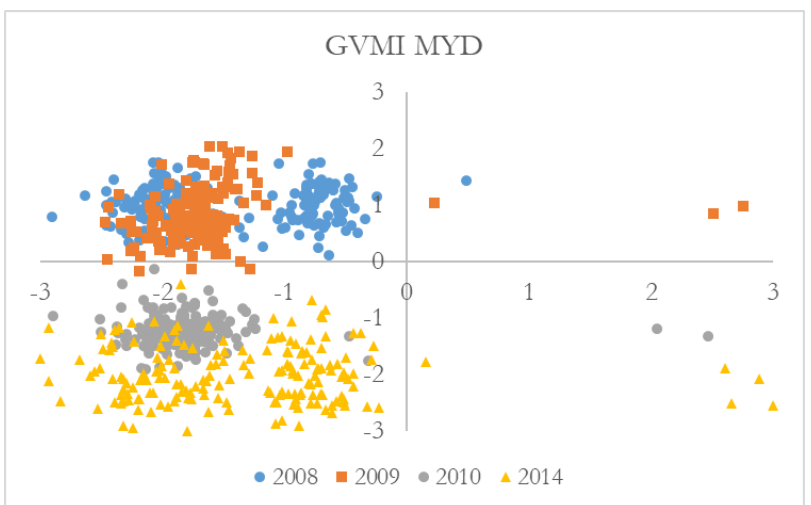

Figura 5: Exemplo de dispersão com separação. O índice está no eixo X e o SPI no eixo Y.

Ao analisar-se a normal climatológica na Figura 7 é possível observar que o mês de fevereiro evidencia a precipitação acumulada, nos anos de 2008 e 2009 acima da média e 2010 e 2014 abaixo. A fase crítica para a cultura da cana de açúcar é o período do mês 11 (novembro) ao mês 2 (fevereiro), por conta disso, o déficit de chuva nesses meses pode influenciar o crescimento da planta e desta forma impactar na cultura criando um evento de seca agrícola.

Foi possível observar que alguns índices espectrais possuíam determinados comportamentos para os anos secos e outro tipo de comportamento para anos úmidos, isso faz com que exista maior relação entre índices e eventos de seca. A Figura 5 mostra que é possível distinguir através do comportamento do índice GVMI os anos secos dos anos úmidos, uma vez que a distinção no eixo y é mais evidente para os pontos amostrais. Já as Figura 6 (índice NDVI) apresenta uma grande mistura entre os anos secos e úmidos e isso pode ser identificado como sendo um problema para a detecção de seca.

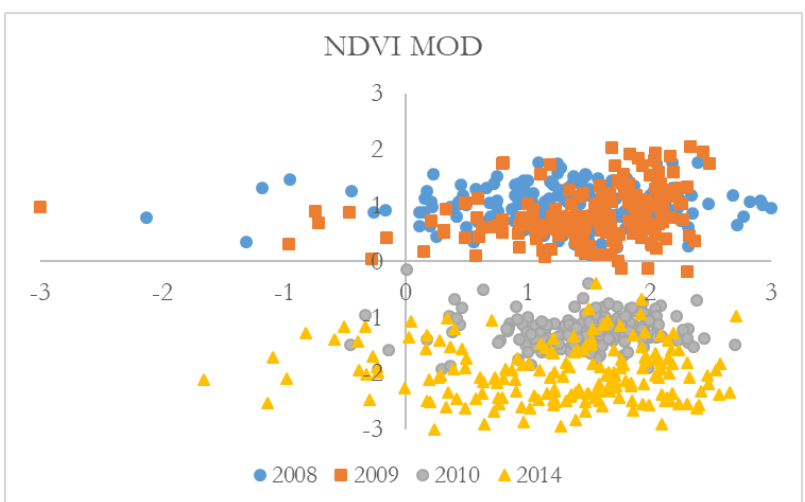

Figura 6: Exemplo de dispersão com confusão. $O$ índice está no eixo $\mathrm{X}$ e o SPI no eixo $\mathrm{Y}$.

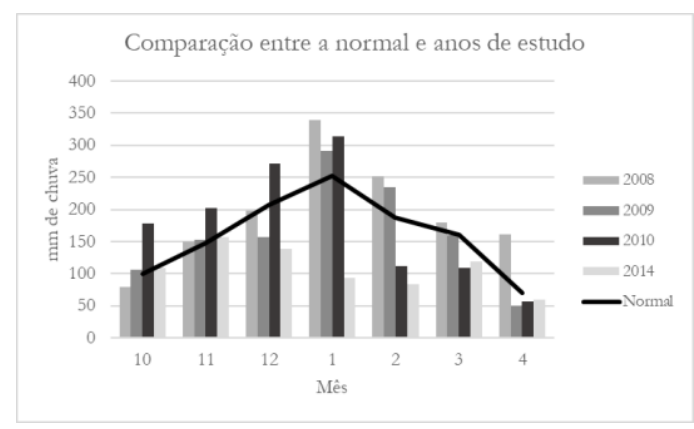

Figura 7: Distribuição da chuva ao longo do ano pela normal climatológica

O ideal seria que os anos úmidos tivessem seus pontos localizados no quadrante positivo, em que os índices espectrais e o SPI são positivos, e os anos secos naquele quadrante em que os índices espectrais e o SPI são negativos. Infelizmente esse efeito não foi notado e pode ser em decorrência de diversos motivos, como por exemplo a diferença de escala entre os dados de entrada do SPI $(25 \mathrm{~km})$ e os dados do sensor MODIS $(0,5 \mathrm{~km})$ que foi utilizado no cálculo dos índices espectrais. Os eventos de seca seguem o mesmo padrão da precipitação, ou seja, é um evento localizado que pode estar presente em vários locais próximos ou não. Desta forma, os dados de $625 \mathrm{~km}^{2}$ não são homogêneos a ponto de representar tudo o que acontece com as culturas em campo. Outro ponto relevante a ser destacado é que diferentes tipos de solo, possuem diferentes capacidades de armazenamento de água e isso reflete na maneira que a cana-de-açúcar sofre com os eventos de seca, desta maneira sob a mesma condição de déficit de precipitação, as plantas podem responder de maneira diferente fisiologicamente e em consequência os índices espectrais apresentam valores diferentes (KIEHL, 1979). Mesmo sem a ocorrência do efeito esperado, foi 
possível identificar que alguns índices separam anos secos dos anos úmidos e essa característica é essencial para a verificação do efeito da seca na cana-de-açúcar quando é necessária uma avaliação em maior escala, como o trabalho se propõe. Foi possível observar eventos de seca (SPI abaixo de zero) durante as safras consideradas normais/chuvosas (2007/08 e 2008/09), isto não ocorreu apenas durante os anos secos. Isso acontece por conta da mudança no período de chuvas devido às mudanças climáticas globais e esse efeito também é prejudicial para uma macroanálise.

Os índices trabalhados possuem comportamentos distintos para a cana-de-açúcar e esses índices variam ao longo das safras. $O$ índice GVMI se destacou por apresentar comportamento desigual entre os anos secos e os úmidos. A Figura 8 mostra a diferença entre as médias mensais dos índices para os anos secos (2009/10 e 2013/14) e os não secos (2007/08 e 2008/09). Para isto, primeiramente foi calculada a média mensal dos índices para os de 2009/10 e 2013/14 (média dos anos secos). Em seguida foi calculada a média mensal dos índices para os de 2007/08 e 2008/09 (média dos anos não secos). Por fim, foi calculada a diferença entre a média dos anos não secos e a média dos anos secos.

É possível notar uma maior amplitude na diferença do índice MSI dos satélites Terra e Aqua. Essa diferença é necessária para que haja um parâmetro de comparação para anos com e sem a ocorrência do evento de seca.

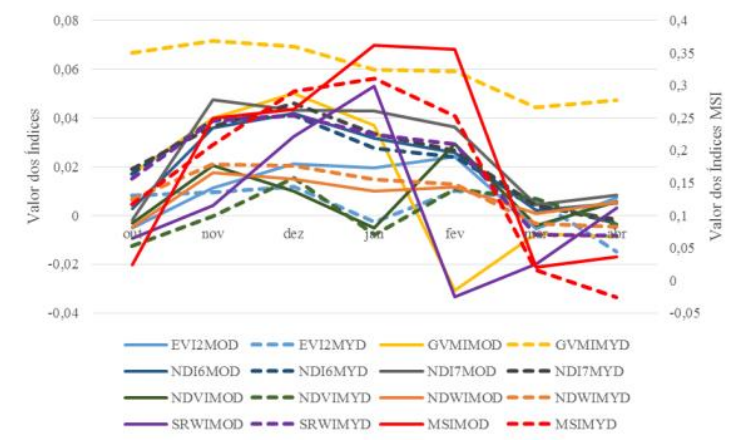

Figura 8: Diferença das médias mensais dos índices em anos secos (2010 e 2014) e não secos (2008 e 2009).

\section{Análise pelo Critério Espacial}

$\mathrm{Na}$ análise através do critério espacial alguns índices espectrais apresentaram correlação significativa (nível de significância $\alpha=5 \%$ ) com o SPI (mensal e trimestral), em alguns pontos do estado de São Paulo. Essa análise foi essencial para ter indicativos de onde cada índice espectral apresenta um melhor desempenho no monitoramento de seca. Na Tabela 2 é possível observar os índices espectrais que mais vezes tiveram correlação e se foi com o SPI MÊS, SPI 3MESES ou ambos.

Tabela 2: Correlações em cada ID do ECMWF

\begin{tabular}{|c|c|c|c|c|c|c|c|}
\hline & 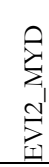 & 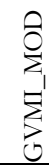 & $\sum_{0}$ & 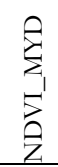 & 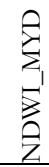 & 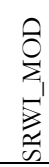 & 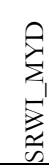 \\
\hline SPI3MESES & 4 & 1 & 9 & 2 & 1 & 1 & \\
\hline SPIMÊS & 1 & 9 & 3 & 4 & 4 & 4 & 5 \\
\hline AMBOS & & & 1 & & 1 & & \\
\hline TOTAL & 5 & 10 & 13 & 6 & 6 & 5 & 5 \\
\hline
\end{tabular}

A correlação entre os índices espectrais e o SPI (mensal e trimestral) aconteceu em poucos pontos do estado. Isto ocorreu devido ao fato dessa análise ter levado em consideração todos os anos safras (2007/08, 2008/09, 2009/10 e 2013/14). Por isso quando os valores dos índices espectrais e dos SPIs foram unidos por localidade e não separados por safra a correlação se mostrou significativa em poucos pontos do estado, uma vez que o caráter de variação espacial das ocorrências de seca foi desprezado.

Porém, o GVMI foi o índice que teve maior número de pontos com correlações significativas com o SPI em todo o estado. Isso é importante para selecionar índices espectrais que representem a seca na maioria do estado. Para determinadas regiões, diversos índices foram significativos, isso ocorreu em grande parte na região norte do estado. Essa região possuía um maior número de amostras, uma vez que a cana é a cultura predominante e, é plantada em áreas mais extensas, permitindo mais amostras de pixel puros de $500 \mathrm{~m}$.

É possível também observar que os índices que mais tiveram correlação foram o GVMI, seguidos pelo NDWI, SRWI e EVI2. De acordo com a literatura (HUNT E ROCK, 1989; CHEN et al., 2005; CACCAMO et al., 2011), índices que utilizam as bandas 6 e 7 do sensor MODIS tem maior potencial de registrar eventos de seca na vegetação e isso foi também observado neste trabalho. Os piores índices foram o MSI MOD, o NDVI MOD e o NDI6 MYD.

Acredita-se que um dos motivos pela falta de correlação em grande parte do estado ocorreu pela janela temporal analisada ser muito grande, ou seja, foram analisados os índices e seu desempenho para todos os anos estudados juntos. Uma análise ano a ano poderia trazer mais índices com alta correlação, mas a intenção da análise foi verificar quais índices tinham a melhor performance durante todo o período analisado. Isso sugere que alguns índices podem ser usados em 
diversas situações que poderão identificar a seca na cana-de-açúcar.

\section{Análise pelo Critério temporal}

Ao analisar temporalmente safra a safra, foi possível observar que alguns índices desempenharam correlação significativa somente nos meses secos. Isso foi importante para identificar qual seria o melhor índice espectral e qual o melhor período para detectar a seca. A fim de isolar efeitos externos, foram agrupados todos os anos-safras para o cálculo da correlação e o resultado está apresentado na Tabela 3.

De acordo com a Tabela 3 é possível observar que o melhor mês para analisar a seca é o mês de fevereiro e utilizando o SPI mensal. Esse mês se mostrou significativo pois como sabe-se os índices são a expressão do comportamento espectral da cana-de- açúcar em determinado momento e as oscilações que expressam a ocorrência de seca são mais notadas em um período que a planta está em desenvolvimento e se espera maior volume de precipitação, como é o caso de fevereiro. O SPI mensal apresentou melhores resultados pois ele registra o cenário pontual naquele mês e não em um período anterior. Como o mês de fevereiro é crítico em termos hidrológicos, o uso do SPI mensal é o mais indicado, pois não sofre influência dos meses anteriores. É ainda necessário ressaltar que a correlação apesar de existir ela obteve valores maiores que 0,5 , em apenas 10 casos. A correlação ocorre quando existe o comportamento semelhante na série de dados, porém é considerada forte quanto mais estiver perto de 1 ou -1 se for uma correlação inversa.

Tabela 3: Correlação entre o SPI e os índices espectrais $(\alpha=0,05)$

\begin{tabular}{|c|c|c|c|c|c|c|c|c|c|c|c|c|c|c|}
\hline & $\begin{array}{l}\text { out } \\
\text { SPI }\end{array}$ & $\begin{array}{c}\text { out } \\
\text { SPI3 }\end{array}$ & $\begin{array}{l}\text { nov } \\
\text { SPI }\end{array}$ & nov & $\begin{array}{l}\text { dez } \\
\text { SPI }\end{array}$ & $\begin{array}{c}\text { dez } \\
\text { SPI3 }\end{array}$ & jan & $\begin{array}{c}\text { jan } \\
\text { SPI3 }\end{array}$ & $\begin{array}{l}\text { fev } \\
\text { SPI }\end{array}$ & $\begin{array}{c}\text { fev } \\
\text { SPI3 }\end{array}$ & $\begin{array}{c}\text { març } \\
\text { SPI }\end{array}$ & $\begin{array}{l}\text { març } \\
\text { SPI3 }\end{array}$ & $\begin{array}{l}\text { abr } \\
\text { SPI }\end{array}$ & $\begin{array}{c}\text { abr } \\
\text { SPI3 }\end{array}$ \\
\hline $\mathrm{D}$ &, $433 *$ & 0,045 & $10^{*}$ & $0,115^{*}$ & 0,037 & $0,123^{*}$ & $-0,024$ & $-0,165^{*}$ & $0,397^{*}$ & $-0,149 *$ & $0,181^{*}$ & $-0,303^{*}$ & $076^{*}$ & $-0,093^{*}$ \\
\hline & & 0 & & & 51 & & & & $0,334^{*}$ & 14 & & $5^{*}$ & 56 & 58 \\
\hline & 381 & $-0,019$ & $0,201^{*}$ & & 0 & $0,152^{*}$ & & & $0,412^{*}$ & $150 *$ & 010 & $-0,319^{*}$ & 0,036 & $-0,108^{*}$ \\
\hline YD & $-0,127^{*}$ & $-0,302 *$ & $-0,006$ & $0,153^{*}$ & $0,405^{*}$ & $-0,248^{*}$ & $0,359 *$ & $-0,524^{*}$ & $0,610^{*}$ & $-0,395^{*}$ & $0,459 *$ & $-0,383^{*}$ & $0,568^{*}$ & $-0,341^{*}$ \\
\hline & $13 *$ & $-0,16$ & $0,199^{*}$ & 0 & 0 , & 0 & $0,141^{*}$ & $-0,2$ & $0,400^{*}$ & $-0,129 *$ & $0,187^{*}$ & $-0,333^{*}$ & $0^{*}$ & $-0,1$ \\
\hline $\mathrm{N}$ & $0,534 *$ & $-0,089^{*}$ & $0,285^{*}$ & $0,158^{*}$ & 0,4 & $0,134^{*}$ & $0,143^{*}$ & $-0,299^{*}$ & $0,408^{*}$ & $-0,039$ & $0,135^{*}$ & $-0,239^{*}$ & $0,085^{*}$ & $-0,094 *$ \\
\hline & 52 & $-0,25$ & & & & 0 & $0,206^{*}$ & -0 , & $0,458^{*}$ & $-0,116^{*}$ & $0,232 *$ & $-0,390^{*}$ & $0,167^{*}$ & $-0,1$ \\
\hline & 0 & $-0,1$ & 0 , & 0 & 0 , & 0, & 0,1 & -0 , & $0,470 *$ & $-0,032$ & $0,156^{*}$ & $-0,274^{*}$ & $0,148^{*}$ & $-0,102 *$ \\
\hline ND & $0,505^{*}$ & $-0,175^{*}$ & $0,194 *$ & $0,148^{*}$ & $0,386^{*}$ & $0,183^{*}$ & $0,138^{*}$ & $-0,289 *$ & $0,391 *$ & $-0,122 *$ & $0,184 *$ & $-0,309^{*}$ & $0,121 *$ & $-0,089 *$ \\
\hline & 0 & $-0,0 \varepsilon$ & 0,2 & 0 & 0 , & 0 & $*$ & -0 & $0,402 *$ & 11 & $4 *$ & $-0,209^{*}$ & 85 & 771 \\
\hline & 0,53 & $-0,001$ & 0,20 & 0 , & -0, & 0,1 & $-0,2$ & 72 & $0,395^{*}$ & $-0,159^{*}$ & $0,197 *$ & $-0,282^{*}$ & $0,154^{*}$ & $-0,109^{*}$ \\
\hline $\mathrm{D}$ & $0,483^{*}$ & $0,163^{*}$ & $0,090 *$ & 0 , & $0,094 *$ & 0 , & $-0,1$ & $0,114 *$ & $0,353^{*}$ & 0,020 & $0,135^{*}$ & $-0,173^{*}$ & 0,0364 & $-0,071$ \\
\hline DD & $401 *$ & $-0,198^{*}$ & $0,185^{*}$ & $39 *$ & $0,412 *$ & $0,109^{*}$ & $0,203^{*}$ & $-0,230^{*}$ & $0,314^{*}$ & $-0,136^{*}$ & $0,212 *$ & $-0,240^{*}$ & $0,191^{*}$ & $-0,075^{*}$ \\
\hline ND & $0,446^{*}$ & $-0,188^{*}$ & $0,197^{*}$ & $0,156^{*}$ & $0,411 *$ & 0,018 & $0,219^{*}$ & $-0,307^{*}$ & $0,414^{*}$ & $-0,062$ & $0,185^{*}$ & $-0,268^{*}$ & $0,133^{*}$ & $-0,093^{*}$ \\
\hline SR & $0,352 *$ & $-0,190^{*}$ & $0,151^{*}$ & 0,052 & $0,422 *$ & $0,094^{*}$ & $0,188^{*}$ & $-0,246^{*}$ & $0,394^{*}$ & $-0,121^{*}$ & $0,249 *$ & $-0,301^{*}$ & $0,219 *$ & $-0,092^{*}$ \\
\hline IYD & $0,444 *$ & $-0,196^{*}$ & $0,205^{*}$ & $0,123^{*}$ & $0,405^{*}$ & 0,014 & $0,225^{*}$ & $-0,317^{*}$ & $0,409^{*}$ & $-0,061$ & $0,170 *$ & $-0,245^{*}$ & $0,129^{*}$ & $-0,097 *$ \\
\hline $\mathrm{SII}$ & 1 & $-0,139^{*}$ & 1 & $0,085^{*}$ & 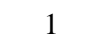 & 0,059 & 1 & $-0,576^{*}$ & 1 & $-0,301^{*}$ & 1 & $-0,494^{*}$ & 1 & $-0,422 *$ \\
\hline SPI3 & $139 *$ & 1 & $0,085^{*}$ & 1 & 0,059 & 1 & $-0,576^{*}$ & 1 & $-0,301 *$ & 1 & $-0,494 *$ & 1 & $-0,422^{*}$ & 1 \\
\hline
\end{tabular}

*Correlações significativas $\operatorname{com} \alpha=0,05$

Os índices que têm maior correlação com o SPI mensal foram o GVMI e o NDI6 ambos do sensor MYD. No trabalho de Caccamo et al. (2011) os autores também identificaram que os índices que usam as bandas 5 e 6 do produto MOD09A tem maior relação com o SPI e identificam a seca em vegetações densas. Apesar de nos outros meses a correlação entre os índices espectrais e o SPI serem significativas, ela foi baixa, inferior a 0,4 .

Diversos autores tiveram resultados que corroboram com os resultados deste trabalho. Bayarjargal et al. (2006) observaram que anomalias nos índices de vegetação tem grande correlação com os índices de seca meteorológica. Zhou et al. (2013) e Jain et al. (2010) mostraram aplicações de índices de seca para o monitoramento dos impactos na vegetação e Du et al. (2013) e Hao et al. (2015) mostram a utilização de sensores diversos integrados como forma de identificar a seca em algumas regiões. Desta forma é possível afirmar que o trabalho obteve resultados importantes para o monitoramento da seca em cana-de-açúcar através de sensores a bordo de satélites. 


\section{CONCLUSÃO}

Utilizando os produtos de refletância do sensor MODIS (MYD e MOD) é possível identificar a seca na cana-de-açúcar. Para uma análise temporal, a data ideal de análise é fevereiro, devido as correlações mais fortes terem ocorrido neste mês. Além disso, é o mês em que existe a maior distinção entre anos secos e anos úmidos.

O SPI que mais se correlacionou com os índices espectrais foi o de período mensal, isso porque a cana-de-açúcar é uma cultura que precisa de água e radiação para desenvolver-se e um período mensal já é suficiente para causar alguma anomalia no crescimento e desenvolvimento da planta, principalmente no período estudado. Portanto, o SPI trimestral não necessariamente reflete as condições na planta, uma vez que o acumulado pode indicar menor ou maior severidade dos fenômenos, porém não com a mesma assertividade temporal.

Os índices espectrais mais significativos foram o GVMI e o NDI6 para a análise temporal, já para a análise espacial ainda é possível destacar o NDWI e o SRWI. Todos esses índices têm em comum o uso das bandas do infravermelho próximo e médio que em conjunto podem evidenciar os efeitos da seca. Já o EVI2, MSI e NDI7 não tiveram correlações com a maioria das amostras do estado, desta forma, pode-se concluir que não são índices indicados para monitorar seca em cana-de-açúcar no estado de São Paulo.

A combinação de índices espectrais com o SPI é uma metodologia que tem grande assertividade, uma vez que o SPI é capaz de captar a seca através da precipitação e os índices espectrais podem verificar se a seca captada realmente afetou o desenvolvimento da cana-de-açúcar.

Para trabalhos futuros alguns pontos podem melhorar a resposta dos índices espectrais para detecção de seca na cana-de-açúcar. Trabalhar com o balanço hídrico para detectar seca ao invés do SPI, melhorar a análise por meses e não por safras e utilizar os índices que apresentaram maior potencial na identificação de seca em imagens dos satélites LANDSAT e PROBA-V para ter uma maior escala de trabalho.

\section{AGRADECIMENTOS}

Agradecemos à Fundação de Amparo à Pesquisa do Estado de São Paulo (Fapesp) pelo suporte financeiro (projeto 2014/17090-5). Aos pesquisadores Dr. Júlio César Dalla Mora Esquerdo (da Embrapa CNPTIA), pela extração das imagens MODIS, ao Dr. Guerric le Maire (do CIRAD), pela confecção da rotina em IDL para cálculo dos índices, ao Dr. Gabriel Blain (do IAC) pelo cálculo do SPI.

\section{REFERÊNCIAS}

ADAMI, M.; RUDORFF, B. F. T.; FREITAS, R. M.; AGUIAR, D. A.; SUGAWARA, L. M.; MELLO, M. P. Remote Sensing Time Series to Evaluate Direct Land Use Change of Recent Expanded Sugarcane Crop in Brazil. Sustainability. p574-585, 2012.

BAYARJARGAL, Y., KARNIELI, A., BAYASGALAN, M., KHUDULMUR, S., GANDUSH, C., \& TUCKER, C. J. A comparative study of NOAA-AVHRR derived drought indices using change vector analysis. Remote Sensing of Environment, 105, p.9-22. 2006.

BLAIN, G. C.; BRUNINI, O. Análise da escala temporal de monitoramento das secas agrícolas e meteorológicas no estado de São Paulo. Revista Brasileira de Meteorologia, v. 22, n. 2, p. 255-261, 2007.

CACCAMO, G., CHISHOLM, L., BRADSTOCK, R. E PUOTINEN, M. Assessing the sensitivity of MODIS to monitor drought in high biomass ecosystems. Remote Sensing of Environment, v. 115, n. 10, p. 2626-2639, 2011.

CEPEA. PIB Cadeias Agropecuárias. Disponível em: $<$ http://www.cepea.esalq.usp.br/pibpec/PIB_Cadeias_2t ri_2015.pdf>. Acesso em: 18 fev. 2016.

CHEN, D. Y.; HUANG, J. F.; JACKSON, T. J. Vegetation water content estimation for corn and soybeans using spectral indices derived from MODIS near- and short-wave infrared bands. Remote Sensing of Environment, v. 98 (2-3), p. 225-236, 2005.

CONAB. Séries Históricas. Disponível em: $<$ http:/ /www.conab.gov.br/conteudos.php?a=1252\&\&P agina_objcmsconteudos=2\#A_objcmsconteudos $>$. Acesso em 3. Jul. 2016. 
DEE, D. P., UPPALA, S. M., SIMMONS, A. J., BERRISFORD, P., POLI, P., KOBAYASHI, S., ANDRAE, U., BALMASEDA, M. A., BALSAMO, G., BAUER, P., BECHTOLD, P., BELJAARS, A. C. M., VAN DE BERG, L., BIDLOT, J., BORMANN, N., DELSOL, C., DRAGANI, R., FUENTES, M., GEER, A. J., HAIMBERGER, L., HEALY, S. B., HERSBACH, H., HÓLM, E. V., ISAKSEN, L., KÅLLBERG, P., KÖHLER, M., MATRICARDI, M., MCNALLY, A. P., MONGE-SANZ, B. M., MORCRETTE, J., PARK, B., PEUBEY, C., DE ROSNAY, P., TAVOLATO, C., THÉPAUT, J. E VITART, F. The ERA-Interim reanalysis: configuration and performance of the data assimilation system. Quarterly Journal of the Royal Meteorological Society, v. 137, n. 656, p. 553-597, 2011.

DOORENBOS, J. \& KASSAM, A.H. Yield response to water. FAO Irrigation and Drainage Paper No. 33. Rome, FAO. 1979.

DU, L.; TIAN, Q., YU, T., MENG, Q., JANCSO, T., UDVARDY, P., \& HUANG, Y. A comprehensive drought monitoring method integrating MODIS and TRMM data. International Journal of Applied Earth Observation and Geoinformation, 23, p.245-253. 2013.

FAO. FAOSTAT database collections. Food and Agriculture Organization of the United Nations. Rome. Disponível em: $<$ http://faostat.org.br $>$. Acesso em 2 de janeiro de 2016.

GUHA-SAPIR D, HOYOIS PH, BELOW R. Annual Disaster Statistical Review 2014: The Numbers and Trends. Brussels: CRED 2015.

HAO, C.; ZHANG, J., \& YAO, F. Combination of multi-sensor remote sensing data for drought monitoring over Southwest China. International Journal of Applied Earth Observation and Geoinformation, 35, p.270-283. 2015

HUNT, E. R.; ROCK, B. N.; NOBEL, P. S. Measurement of leaf relative water content by infrared reflectance. Remote Sensing of Environment, v. 22 (3), p. 429-435, 1987.

IBGE. Produção Agrícola Municipal - Disponível em: <http://sidra.ibge.gov.br>. Acesso em 30 jul. 2016.
INTERGOVERNMENTAL PANEL ON CLIMATE CHANGE (IPCC). WGII AR5: Climate Change 2014: Impacts, Adaptation, and Vulnerability: Summary for Policymakers, 2014. Disponível em: http://www.ipcc.ch/report/ar5/wg2/ Acesso em: Abril, 2014.

JAIN, S.K.; KESHRI, R., GOSWAMI, A., \& SARKAR, A. Application of meteorological and vegetation indices for evaluation of drought impact: a case study for Rajasthan, India. Natural hazards, 54, p.643-656. 2010.

KIEHL, E.J. Manual de edafologia. São Paulo, Agronômica Ceres, p.262, 1979.

KOEHLER, P. H., MOORE, P. H., JONES, C. A., DELA CRUZ, A. E MARETZKI, A. Response of Drip-Irrigated Sugarcane to Drought Stress1. Agronomy Journal, v. 74, n. 5, p. 906, 1982.

MACHADO, P. G., PICOLI, M. C. A., TORRES, L. J., OLIVEIRA, J. G. E WALTER, A. The use of socioeconomic indicators to assess the impacts of sugarcane production in Brazil. Renewable and Sustainable Energy Reviews, v. 52, p. 1519-1526, 2015.

MAMADOU, O., COHARD, J. M., GALLE, S., AWANOU, C. N., DIEDHIOU, A., KOUNOUHEWA, B. E PEUGEOT, C. Energy fluxes and surface characteristics over a cultivated area in Benin: daily and seasonal dynamics. Hydrol. Earth Syst. Sci., v. 18, n. 3, p. 893-914, 2014.

MANZATTO, C.V.; ASSAD, E.D., BACCA, J.F.M., ZARONE, M.J., PEREIRA, S.E.M., 2009. Zoneamento agroecológico da cana-de-açúcar. Expandir a produção preservar a vida, garantir o futuro. Rio de Janeiro (EmbrapaSolos). Presentation available at 〈http://www.cop15.gov.br/enUS/indexc576.html?page=panorama/ agro-ecologicalzoning-of-sugarcane). Disponível em: $<$ http://www.agricultura.gov.br/arq_editor/anuario_a groenergia_WEB_small.pdf $>$. Acesso em: 4 fev. 2016. 
MARIN, F. R., JONES, J. W., SINGELS, A., ROYCE, F., ASSAD, E. D., PELlEGRINO, G. Q. E JUSTINO, F. Climate change impacts on sugarcane attainable yield in southern Brazil. Climatic Change, v. 117, n. 1-2, p. 227-239, 2012.

MCKEE, T. B.; DOESKEN, N. J.; KLEIST, J. The relationship of drought frequency and duration to time scales. Anais... Eighth Conference on Applied Climatology, American Meteorological Society, Anaheim, CA, p. 179-184. 17-22 janeiro, 1993.

MCKEE, T. B.; DOESKIN, N. J.; KLEIST, J. Drought monitoring with multiple time scales. Anais... Ninth Conference on Applied Climatology, American Meteorological Society, Boston, MA, p. 233-236. 15-20 jan, 1995.

NAGLER, P., GLENN, E., NGUYEN, U., SCOT'T, R. E DOODY, T. Estimating Riparian and Agricultural Actual Evapotranspiration by Reference Evapotranspiration and MODIS Enhanced Vegetation Index. Remote Sensing, v. 5, n. 8, p. 3849-3871, 2013.

ORLOWSKY, B.; SENEVIRATNE, S. Global changes in extreme events: regional and seasonal dimension. Climatic Change, v. 110, n. 3-4, p. 669-696, 2011.

PALMER, W. C. Meteorological drought. Research Paper, vol. 45. U.S. Department of Commerce Weather Bureau, Washington, DC. 1965.

PALMER, T.; RÄISÄNEN, J. Quantifying the risk of extreme seasonal precipitation events in a changing climate. Nature, v. 415, n. 6871, p. 512-514, 2002.

PEEL, M.; FINLAYSON, B.; MCMAHON, T. Updated world map of the Köppen-Geiger climate classification. Hydrology and Earth System Sciences Discussions, v. 4, n. 2, p. 439-473, 2007.

RHEE, J.; IM, J.; CARBONE, G. Monitoring agricultural drought for arid and humid regions using multi-sensor remote sensing data. Remote Sensing of Environment, v. 114, n. 12, p. 2875-2887, 2010.
SON, N., CHEN, C., CHEN, C., CHANG, L. E MINH, V. Monitoring agricultural drought in the Lower Mekong Basin using MODIS NDVI and land surface temperature data. International Journal of Applied Earth Observation and Geoinformation, v. 18, p. 417-427, 2012.

TUCKER, C. Red and photographic infrared linear combinations for monitoring vegetation. Remote Sensing of Environment, v. 8, n. 2, p. 127-150, 1979.

UNICA - UNIÃO DA INDÚSTRIA DE CANA-DEAÇÚCAR - POR SAFRA. Unicadata. Disponível em: $<$ http://www.unicadata.com.br/ >. Acesso em: 4 fev. 2016.

USGS - U.S. Geological Service. MODIS Products Table. Disponível em: < https://lpdaac.usgs.gov/dataset_discovery/modis/mo dis_products_table>. Acesso em: 4 fev. 2016.

WALTER, A., DOLZAN, P., QUILODRÁN, O., DE OLIVEIRA, J. G., DA SILVA, C., PIACENTE, F. E SEGERSTEDT, A. Sustainability assessment of bioethanol production in Brazil considering land use change, GHG emissions and socio-economic aspects. Energy Policy, v. 39, n. 10, p. 5703-5716, 2011.

WALTER, A., GALDOS, M. V., SCARPARE, F. V., LEAL, M. R. L. V., SEABRA, J. E. A., DA CUNHA, M. P., PICOLI, M. C. A. E DE OLIVEIRA, C. O. F. Brazilian sugarcane ethanol: developments so far and challenges for the future. WIREs Energy Environ, v. 3, n. 1, p. 70-92, 2013.

WU, Z., LIN, Q., LU, G., HE, H. E QU, J. J. Analysis of hydrological drought frequency for the Xijiang River Basin in South China using observed streamflow data. Nat Hazards, v. 77, n. 3, p. 1655-1677, 2015.

ZHANG, L., SUN, X., WU, T. E ZHANG, H. An Analysis of Shadow Effects on Spectral Vegetation Indexes Using a Ground-Based Imaging Spectrometer. IEEE Geoscience and Remote Sensing Letters, v. 12, n. 11, p. 2188-2192, 2015. 
ZHOU, L.; WU, J., ZHANG, J., LENG, S., LIU, M., ZHANG, J., ZHAO, L., ZHANG, F., \& SHI, Y. The integrated surface drought index (ISDI) as an indicator for agricultural drought monitoring: theory, validation, and application in Mid-Eastern China. Selected Topics in Applied Earth Observations and Remote Sensing, IEEE Journal of, 6, p.1254-1262. 2013.

ZSCHEISCHLER, J., MICHALAK, A. M., SCHWALM, C., MAHECHA, M. D., HUNTZINGER, D. N., REICHSTEIN, M., BERTHIER, G., CIAIS, P., COOK, R. B., ELMASRI, B., HUANG, M., ITO, A., JAIN, A., KING, A., LEI, H., LU, C., MAO, J., PENG, S., POULTER, B., RICCIUTO, D., SHI, X., TAO, B., TIAN, H., VIOVY, N., WANG, W., WEI, Y., YANG, J. E ZENG, N. Impact of large-scale climate extremes on biospheric carbon fluxes: An intercomparison based on MsTMIP data. Global Biogeochem. Cycles, v. 28, n. 6, p. 585-600, 2014. 